\title{
Sustentabilidad social del sistema agrícola de maíz amarillo duro en el Valle de Pativilca - Lima
}

\author{
Social Sustainability of the Agricultural System of Hard Yellow \\ Corn in the Pativilca Valley - Lima
}

Fernando Carlos Bravo Martínez ${ }^{1 *}$, Percy Zorogastua Cruz ${ }^{1}$, Rember Pinedo Taco ${ }^{1}$

\section{RESUMEN}

El cultivo de maíz amarillo duro (MAD) constituye la principal fuente de ingresos para los productores en el valle de Pativilca, por lo tanto, esta actividad es determinante en la situación socioeconómica de esos trabajadores. Por esta razón se realizó la investigación con el objetivo de definir el nivel de sostenibilidad social del cultivo de MAD y su repercusión en los modos de vida de los agricultores que lo practican. Los datos analizados provienen de 73 encuestas realizadas a productores de MAD de tres comisiones de regantes. Los valores de los indicadores y subindicadores evaluados resultaron sostenibles, al igual que el Indicador Social (IS), por tener un valor de 3,54, mayor a 3 definido como el umbral mínimo de sostenibilidad de acuerdo a la metodología de análisis multidimensional utilizada. A nivel de comisiones de regantes el mayor valor de IS lo alcanzó la Comisión de Riego de Araya, en segundo lugar la Comisión de Riego de Potao y tercero la Comisión de Riego de Vinto. Si bien las tres comisiones de regantes superan el valor del umbral mínimo establecido, se ubican en el rango de sostenibilidad débil, y factores bióticos, abióticos y el comportamiento del mercado pueden afectaresta condición.

Palabras clave: indicador, integración social, asistencia técnica, capacitación, sistema de producción

\begin{abstract}
The cultivation of hard yellow corn (MAD) is the main source of economic income for producers in the valley of Pativilca, therefore, its cultivation is intensive. For this reason, the research was carried out with the objective of defining the level of social sustainability of the MAD crop and its repercussion on the ways of life of the farmers who practice it. The analyzed data come from 73 surveys made to producers of MAD three irrigation commissions. The values found for the indicators and sub-indicators analyzed were sustainable and the Social Indicator (IS), having a value of 3.54 greater than 3, defined as the minimum sustainability threshold according to the multidimensional analysis methodology used, was sustainable. At the irrigation committee level, the highest value of IS reached the irrigation commission of Araya, in second place the Irrigation Commission of Potao and thirdly the Irrigation Commission of Vinto. However, although the three irrigation commissions exceed the minimum threshold value established, they fall within the range of weak sustainability and biotic, abiotic factors and market behavior can affect their sustainability.

Key words: indicator, social integration, technical assistance, training, production system
\end{abstract}

\section{Introducción}

La producción mundial del cultivo de maíz amarillo duro (MAD) bordea los 1.070 millones de toneladas, y los principales países productores son Estados Unidos, China y Brasil, Argentina, México, Ucrania, India e Indonesia, que acumulan en conjunto, un poco más del $50 \%$ de toda la producción. Según información del Departamento de Agricultura de los Estados Unidos (USDA), este país continuaría liderando la producción mundial con 372 millones de toneladas (34\% del total mundial), seguido de China con 256 millones de toneladas (23\% del total mundial) y Brasil con 94,5 millones de toneladas $(8,6 \%$ del total mundial). Estos países concentrarían 2 tercios de la producción mundial (MINAGRI, 2019).

La producción nacional del cultivo de maíz amarillo duro (MAD) alcanza a 277 mil hectáreas (Huamanchumo, 2013). Para la campaña del año 2018 la producción nacional de maíz amarillo duro se ubicó en 256. 163 ha (MINAGRI, 2019). En la Costa Central, específicamente en Barranca, el cultivo de maíz amarillo duro cubre unas 6.000

\footnotetext{
1 Universidad Nacional Agraria La Molina (UNALM). Lima, Perú.

* Autor por correspondencia: fbravom@hotmail.com; 20120487@lamolina.edu.pe
} 
hectáreas en promedio. Al respecto, el INEI (2015) reporta 8,550 ha para la campaña 2013-2014; mientras que en la campaña 2015-2016 la superficie cultivada se incrementó a 9,477 ha y en la campaña 2015-2016 se redujo a 4,368 ha. La importancia social del cultivo de maíz radica en la cantidad de productores que se dedican a esta labor y, por ende, en la generación de mano de obra y la seguridad alimentaria (CEPES, 2018). Según el INEI (2015), el $31,9 \%$ de la población económicamente activa (PEA) del distrito de Barranca se dedica a la agricultura. El MAD es un cultivo sensible, no solo porque representa el principal insumo para la industria avícola y porcina, sino también por ser una fuente esencial de ingresos y empleo para los pequeños y medianos productores ubicados en los valles costeros de la costa y selva (Fernández, 2016; CEPES, 2018). Pese a su alto impacto económico por la existencia de un arancel de $12 \%$ sobre el maíz norteamericano, el Perú importa más de un millón de toneladas de MAD. El Sistema Peruano de Franja de Precios se creó para proteger la producción agropecuaria nacional de la competencia de las importaciones mediante aranceles específicos variables. En este contexto, las consecuencias en términos económicos, ambientales y principalmente sociales son inciertas para la producción doméstica de casi un millón de toneladas, que da empleo aproximadamente a 70 mil trabajadores (Fernández, 2016).

Con respecto al tema social, en la provincia de Barranca, el Índice de Desarrollo Humano (IDH) que integra los parámetros vida larga y saludable, educación y nivel de vida digno, es de 0,68 y en el distrito de Pativilca 0,65, siendo el más bajo a nivel provincial (MPB 2015). Precisamente, el marco conceptual de esta investigación prima el desarrollo humano sostenible, el cual debe permitir que la población tenga acceso a servicios básicos de calidad, servicios de fortalecimiento de capacidades a través de la capacitación y asistencia técnica, y espacios democráticos para organizarse colectivamente (MPB 2015). Este es el desafío de los 17 objetivos de desarrollo sostenible para el 2030 acordados por las Naciones Unidas, de los cuales ocho se encuentran vinculados a las áreas de la salud, educación, derecho a la organización, equidad de género, acceso a bienes y servicios, entre las más destacables (ONU 2015).

Las políticas públicas de los gobiernos locales buscan que todas las personas tengan igualdad de oportunidades para desarrollarse, lo que implica el acceso a servicios básicos de calidad, en particular educación, salud, agua y desagüe, electricidad, telecomunicaciones, vivienda y seguridad ciudadana. Según las propuestas de los gobiernos locales, el acceso universal a servicios de calidad y la seguridad alimentaria son esenciales para superar la pobreza y garantizar igualdad de oportunidades para todos (MPB, 2015). Al respecto, la FAO indica que para ser sostenible, la agricultura debe satisfacer las necesidades de las generaciones presentes y futuras de sus productos y servicios, garantizando al mismo tiempo la rentabilidad, la salud del medio ambiente y la equidad social y económica (FAO, 2016). Así el desarrollo agrícola sostenible contribuye a garantizar la equidad y responsabilidad social de la agricultura y los sistemas alimentarios.

En el aspecto organizativo, el valle de Pativilca cuenta con la Junta de Usuarios Pativilca del distrito de riego Barranca, organización responsable de mantener, planificar y controlar la provisión de agua para los distintos sistemas de cultivos y crianzas de 16 comisiones de regantes (ANA, 2015; MINAGRI, 2008). El valle se encuentra en la Cuenca Hidrográfica del río Pativilca y cuenta con una superficie de 25,443 ha Bajo Riego y 7,721 usuarios en el régimen de Permiso. En este contexto se trata de encontrar el mayor significado de la palabra "sostenibilidad", vinculándolo con objetivos concretos como desarrollo humano, sociedad sostenible y agricultura sostenible (Reiche y Carls, 1996). El factor humano es el principal dinamizador de los agroecosistemas, por lo tanto, en términos de equidad, no solo es importante el desarrollo económico y el cuidado del medio ambiente, sino también la mejora de los servicios y la asistencia técnica, así como facilitar los procesos de organización con enfoque territorial y sistémico (FAO, 2014). El concepto de sustentabilidad es complejo en sí mismo porque implica cumplir, simultáneamente, con varios objetivos: productivos, ambientales, sociales, culturales, económicos (Sarandón, 2002; Sarandón y Flores, 2014; FAO 2014; ONU 2015). Por tal razón, se propone analizar el aspecto social con un enfoque multidimensional para definir los niveles de sostenibilidad de los productores dedicados al cultivo de MAD.

\section{Materiales y Métodos}

La investigación se realizó en el valle de Pativilca, provincia de Barranca, Región Lima, ubicada entre 
los paralelos $10^{\circ} 00^{\prime}$ y $11^{\circ} 00^{\prime}$ de latitud sur y entre los meridianos $76^{\circ} 46^{\prime}$ y $77^{\circ} 57^{\prime}$ 'de longitud oeste (Figura 1). Esta zona presenta un clima templado y desértico. Respecto a la precipitación, registra un nivel muy escaso, aproximadamente $7 \mathrm{~mm} / \mathrm{año}$. El abastecimiento hídrico proviene del río Pativilca.

La etapa de investigación fue no experimental, descriptiva y explicativa. En esta fase se evaluó la sostenibilidad de las unidades o parcelas productoras de MAD en la zona central del distrito de Pativilca. La delimitación del área en estudio fue producto de un análisis y diagnóstico territorial previo de la cuenca, el cual determinó la identificación de la unidad geográfica-económica de interés que resultó ser la cuenca media y baja del río Pativilca, constituida por un conjunto de condiciones homogéneas físicas, económicas y sociales (Ayora, 2015).

De una población de 360 productores de maíz amarillo duro se obtuvo una muestra irrestricta aleatoria $(\mathrm{n}=73)$, utilizando la fórmula de Scheaffer et al. (1987). Luego, de acuerdo al modelo de Márquez (2015) y Pinedo et al. (2018), se seleccionó a los agricultores mediante muestreo sistemático de tres comisiones de regantes del valle de Pativilca.

La evaluación se realizó con el uso de indicadores elaborados para la dimensión social y de forma comparativa entre las tres comisiones de regantes a las que pertenece cada sector. Ha servido para determinar los niveles de sostenibilidad de la producción de MAD en el ámbito de estudio. La investigación se hizo aplicando el enfoque cualitativo (Flores y Sarandón, 2006) y la propuesta metodológica de análisis multivariado de Sarandón (2002), basada en la utilización de indicadores socioeconómicos y ambientales.

\section{Construcción de indicadores}

El desarrollo y uso de indicadores es una herramienta adecuada y flexible para evaluar tendencias, establecer diferencias entre fincas y detectar los puntos críticos de manejo de recursos para el logro de una agricultura sustentable (Flores y Sarandón, 2004). Se eligieron cuatro indicadores (nivel de satisfacción de las necesidades básicas, financiamiento de los sistemas de producción de MAD, la integración social y la valoración de capacitación y asistencia técnica) y nueve subindicadores (Tabla 1) fáciles de obtener e interpretar, que brindan la información necesaria y que permiten detectar tendencias en el ámbito de estudio de Sarandón (2002). Se consideró la importancia de la vivienda con respecto a su nivel de tenencia y distancia a los centros de producción. Otro subindicador importante fueron los niveles de educación que pueden ser determinantes en los niveles de adopción de tecnologías (Pinedo et al., 2017; CYMMYT, 2003), asimismo la calidad de salubridad del productor. Elcomponente de calidad de vías de acceso a la parcela del productor incide en los costos indirectos de producción y muy relacionado con este subindicador está el tema de la disponibilidad de movilidad de los agricultores tanto para trasladarse a sus fincas como para transportarr insumos y cosechas. La infraestructura vial tiene un carácter geo-económicos importante para el desarrollo económico y social de la provincia. No solo permite el traslado de bienes sino que también forma áreas de intercambio de personas (MPB, 2015). Para el indicador de integración social se evaluó con el subindicador de niveles de asociatividad, es decir, los niveles de organización
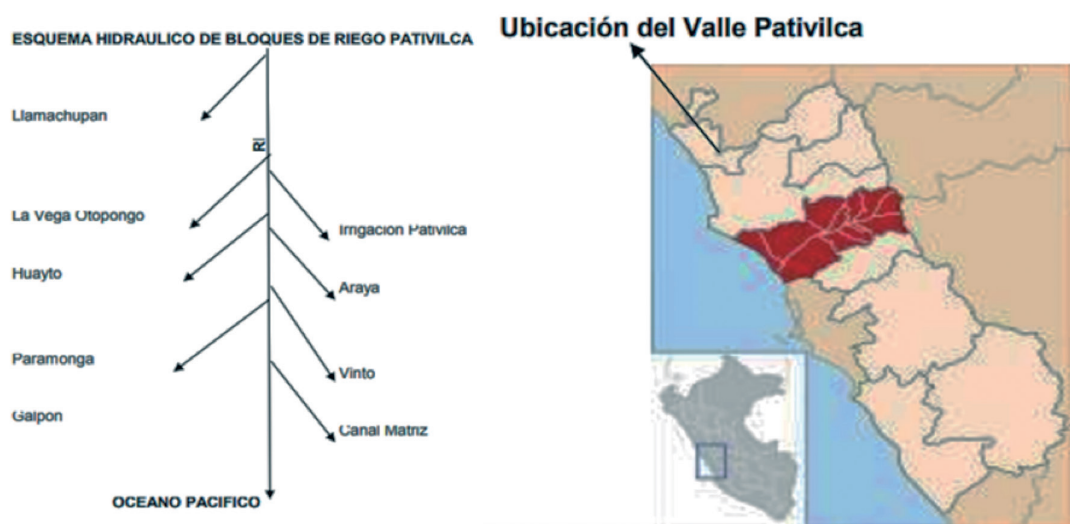

Figura 1. Mapa de ubicación del valle de Pativilca, Provincia de Barranca. Fuente MPB (2015). 
Tabla 1. Indicadores y subindicadores de sostenibilidad social.

\begin{tabular}{|c|c|c|}
\hline Indicador & Subindicador & Escalas de valor de los indicadores \\
\hline \multirow{6}{*}{$\begin{array}{l}\text { A. Necesidades } \\
\text { básicas }\end{array}$} & A1. Nivel de educación & (5) Universitario; (4) Técnico; (3) Secundaria; (2) Primaria; (1) Ninguna \\
\hline & A2. Vivienda & $\begin{array}{l}\text { (5) Material noble, muy buena; (4) Material noble, buena; (3) Material adobe, } \\
\text { buena; (2)Material adobe regular; (1) Material adobe mala }\end{array}$ \\
\hline & $\begin{array}{l}\text { A3. Servicios básicos de la } \\
\text { vivienda }\end{array}$ & $\begin{array}{l}\text { (5) Servicios de agua, desagüe, luz e internet; (4): Servicios de agua, desagüe, luz } \\
\text { sin internet ; (3) Servicios de agua, ni desagüe ni luz; (2) Agua, sin desagüe y sin } \\
\text { luz; (1) Sin ningún servicio básico }\end{array}$ \\
\hline & A4. Calidad de servicios de salud & $\begin{array}{l}\text { (5) Muy buena, equipamiento y servicios eficientes; (4) Buena, equipado, servicios } \\
\text { adecuados, (3) Regular, equipado pero con servicios inadecuados; (2) Mala, muy } \\
\text { mala, equipos obsoletos, mala calidad de servicios; (1) Muy mal: sin equipos, solo } \\
\text { servicios de atención primaria. }\end{array}$ \\
\hline & $\begin{array}{l}\text { A5. Calidad de vías de acceso a } \\
\text { su finca }\end{array}$ & $\begin{array}{l}\text { (5) Muy buenas condiciones; (4); Buena; (3); Regular; (2) Mala; (1); Muy malas } \\
\text { condiciones. }\end{array}$ \\
\hline & $\begin{array}{l}\text { A6. Disponibilidad/estado de } \\
\text { movilidad }\end{array}$ & $\begin{array}{l}\text { 5) Propia en buen estado, 4) propia regular estado; 3) propia deteriorada; 2) alquiler } \\
\text { 1) no tiene ni alquila. }\end{array}$ \\
\hline \multirow{3}{*}{$\begin{array}{l}\text { B. Nivel de } \\
\text { satisfacción e } \\
\text { integración social }\end{array}$} & $\begin{array}{l}\text { B1. Nivel de satisfacción del } \\
\text { productor }\end{array}$ & $\begin{array}{l}\text { (5): Muy satisfecho; (4): satisfecho; (3): Medianamente satisfecho; (2): Muy poco } \\
\text { satisfecho; (1): insatisfecho. }\end{array}$ \\
\hline & B2. Lugar de residencia & $\begin{array}{l}\text { (5) Parcela o fundo; (4) Centro poblado muy cercano a su parcela; (3) Centro } \\
\text { poblado alejado de su parcela; (2) Ciudad muy cercana a su parcela; (1) Ciudad } \\
\text { muy lejos de su parcela. }\end{array}$ \\
\hline & B3. Integración social & (5) Excelente (4) Muy buena; (3) Buena; (2) Regular; (1) Nula. \\
\hline \multirow{3}{*}{$\begin{array}{l}\text { C. Asistencia técnica/ } \\
\text { capacitación }\end{array}$} & $\begin{array}{l}\text { C1. Calidad de asistencia técnica } \\
\text { y capacitación }\end{array}$ & (5) Excelente; (4) Muy buena; (3) Buena; (2) Regular; (1) Nula, no le sirve. \\
\hline & C2. Oferta tecnológica & $\begin{array}{l}\text { (5)Muy buena, variedades/tecnologías de manejo de MAD; (4) Buena, } \\
\text { variedades insumos; (3) Regular poca oferta de variedades y métodos } \\
\text { de producción; (2) Mala, falta semillas de calidad, escasos métodos de } \\
\text { producción; (1) Muy mala, poca oferta tecnológica. }\end{array}$ \\
\hline & $\begin{array}{l}\text { C3. Conocimiento y manejo de } \\
\text { agroquímicos }\end{array}$ & $\begin{array}{l}\text { (5): Muy buena, manejo excelente de uso efectividad y nivel de toxicidad (tiene } \\
\text { estudios); (4) Buena: conoce muy bien su efectividad y nivel de toxicidad; } \\
\text { (3): Regular, conoce bien o adecuadamente los productos (2): Mala, conoce } \\
\text { regularmente los productos; (1) Muy mal: Conoce muy poco usa por indicaciones } \\
\text { del técnico, asesor o vecino. }\end{array}$ \\
\hline
\end{tabular}

de los productores para participar en las compras y ventas colectivas. Finalmente se analizó el indicador de asistencia técnica y capacitación con la percepción de los agricultores sobre la calidad y oportunidad de asistencia técnica para mejorar la productividad y competitividad de los productores de MAD.

Para comparar las unidades de producción (parcelas) y facilitar el análisis de los indicadores y subindicadores identificados para la dimensión social, se estandarizaron los datos modificando la propuesta original de Sarandón (2002) de 0 a 4 a una escala de 1 a 5 . En este caso fue 1 el valor crítico, 3 el umbral mínimo y 5 el umbral o valor máximo de sostenibilidad (Pinedo et al., 2018; Meza y Julca, 2015). Esto facilitó la integración de varios indicadores de distinta naturaleza, en otros más sintéticos o robustos (Sarandón, 2002). La utilización de razones matemáticas ha permitido estandarizar los valores absolutos y optimizar el manejo de los datos, además de una mejor comprensión de la dinámica propia de los indicadores calculados (García y Calle, 1998).

Posteriormente, para determinar el Indicador Social (IS), los subindicadores de cada indicador fueron ponderados con la siguiente relación matemática: $\mathrm{IS}=[(\mathrm{A} 1+2 * \mathrm{~A} 2+2 * \mathrm{~A} 3+2 * \mathrm{~A} 4+\mathrm{A} 5$ $+\mathrm{A} 6) 9+(\mathrm{B} 1+\mathrm{B} 2+\mathrm{B} 3) / 3+2((\mathrm{C} 1+\mathrm{C} 2+\mathrm{C} 3) / 3) / 4]$. 
Los indicadores fueron ponderados multiplicando el valor de la escala por un coeficiente de acuerdo a la importancia relativa de cada variable respecto a la sustentabilidad. Este coeficiente multiplica tanto el valor de las variables que forman el indicador, como el de los indicadores, para construir indicadores de mayor nivel o índices (Meza y Julca, 2015). La ponderación se validó por discusión y consenso entre técnicos, expertos y agricultores líderes que participaron en el trabajo, posibilitando la evaluación y definición de indicadores en forma participativa (Pinedo et al., 2017). Dentro de este objetivo, se considerarán de mayor peso los subindicadores de acceso a la educación, calidad de servicios de salud, servicios básicos y nivel de asistencia técnica y capacitación.

\section{Resultados y Discusión}

\section{Aspectos socioeconómicos y organizativos de los agricultores en el valle de Pativilca}

El valle de Pativilca se ubica en los distritos políticos de Barranca, Supe, Pativilca, Paramonga en la provincia de Barranca, Cochas, Acas, Manas, de la provincia de Ocros, y Cajatambo, en la cuenca del río Pativilca. Pertenece administrativamente al Sector de Riego Barranca, del distrito de Riego Barranca. En 2008 contaba con 15 comisiones de regantes y para el año 2017 se incrementó a 16 (ANA, 2008; MINAGRI, 2008). Existen un total de 5.977 usuarios y 7.481 predios. Dentro del Programa de Formalización de los Derechos de Uso de Agua, se han establecido nueve (9) bloques de riego. Las áreas regadas en el valle de Pativilca son abastecidas por las aguas provenientes del río del mismo nombre, y de manera complementaria con agua subterránea.

Los diez cultivos de mayor importancia en el valle de Pativilca son caña de azúcar $(47,96 \%)$, maíz amarillo duro, algodón tanguis, camote amarillo, papa perricholi, maíz chala, mandarina malvácea, mandarina, papa canchán y manzana Israel. Ocupan una superficie de 24.983 ha.

La optimización de la gestión de los recursos hídricos implica una disminución de los conflictos sociales en torno a la justa distribución y uso del agua (MINAGRI, 2008).

La Figura 2A muestra que los niveles de educación primaria y secundaria prevalecen con $38,36 \%$ y $47,90 \%$. Según el MPB (2015) existe un $10 \%$ de población que no tiene ningún nivel de educación. El 53\% de los productores poseen viviendas de buena calidad con material noble, mientras que el $19,18 \%$ y $27,40 \%$ tienen viviendas de material de adobe de regular y buena calidad (Figura 2B).

El 98,63\% de los servicios de capacitación son realizados por los proveedores de agroquímicos (Figura 3A). El 84,73 de los productores perciben que la calidad de los servicios de capacitación es regular, el 13,70 asegura que es buena y $1,37 \%$ califica de muy buenos los servicios del sector público (Figura 3B). El 86,30\% conoce bien las características y forma de aplicación de los pesticidas, mientras que el 10,96\% lo conoce muy bien (Figura 3B). El proceso de innovación y tecnificación de la gestión de los sistemas
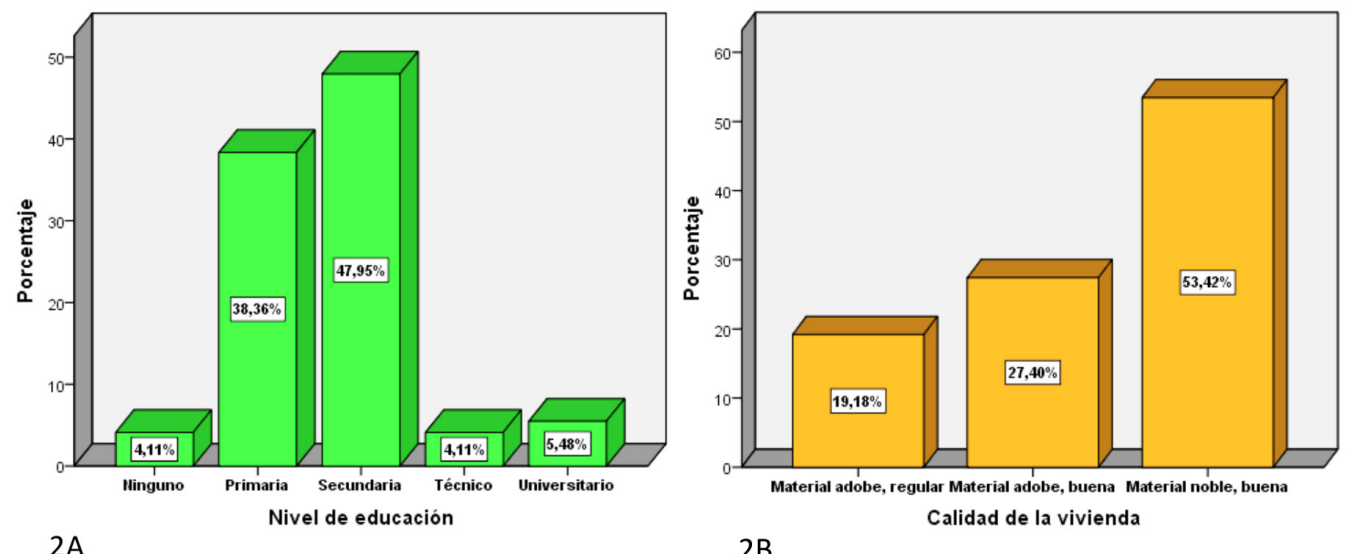

$2 \mathrm{~A}$

Figura 2. Nivel de educación y calidad de la vivienda de los productores de MAD en $\%$. 

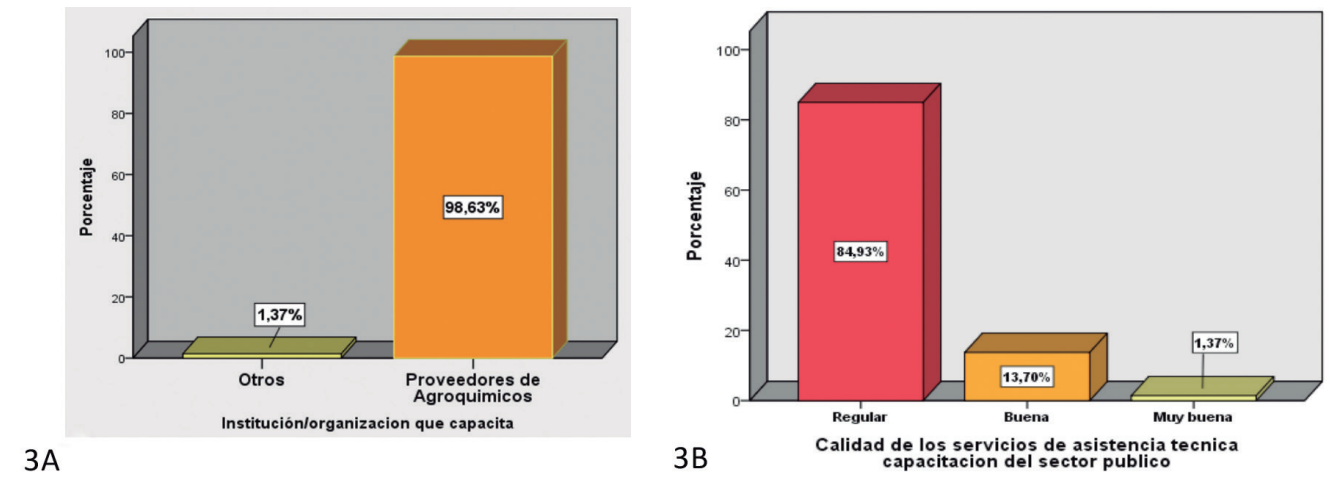

Figura 3. Institución que brinda servicios de capacitación y calidad de los servicios de asistencia técnica.

de producción requiere planificación, uso de metodologías y protocolos para realizar acciones de transferencia de tecnologías y el monitoreo y evaluación de los niveles de adopción de tecnologías que pueden ser afectados por el nivel de educación de los productores (CINMYT, 2003; Pinedo et al., 2018). Aun si fueran ideales los niveles de educación, actualmente los servicios de capacitación y asistencia técnica que realiza el INIA no atienden ni el 1\% de la demanda de capacitación de los 2.164 .000 productores del país (INEI, 2013).

Con respecto al conocimiento del uso correcto de plaguicidas, el $86,30 \%$ conoce bien sus características y forma de aplicación , mientras que el 10,96\% lo conoce muy bien (Figura 4). El SENASA, órgano adscrito al MINAGRI, designado como Autoridad en Sanidad Vegetal, promueve campañas de manejo de agroquímicos en alianza con las empresas privadas; sin embargo, las empresas privadas, los organismos no gubernamentales y sobre todo los proveedores de agroquímicos tienen

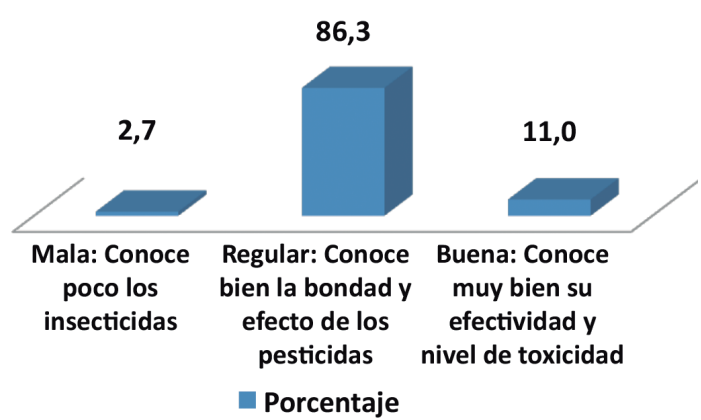

Figura 4. Nivel de conocimiento sobre pesticidas agrícolas en porcentaje. la mayor participación en servicios de capacitación y asistencia técnica directa (INEI, 2013).

\section{Análisis de sostenibilidad}

De acuerdo al protocolo de análisis multicriterio, utilizando indicadores y basado en la propuesta metodológica de Sarandón (2002), el indicador de satisfacción de necesidades básicas alcanzó un valor de 3,44. Los subindicadores que aportaron al valor registrado fueron calidad de vivienda, disponibilidad de servicios básicos, calidad de los servicios de salud, disponibilidad de movilidad y estado de conservación de las vías de acceso a sus unidades de producción. Mientras que la sumatoria de los subindicadores de nivel de satisfacción e integración social fue de 3,89 y el indicador de capacitación y nivel de asistencia técnica 3,42 (Figura 3). De acuerdo a la fórmula matemática elaborada, el IS resultó en 3,58 , valor que es sostenible según la propuesta metodológica de Sarandón (2002). Sin embargo, de acuerdo a los análisis de sostenibilidad realizados por Pinedo et al. (2018) y Meza y Julca (2015), este valor califica como sostenibilidad débil y factores internos (nivel de organización, cohesión familiar) y externos como el comportamiento del clima y mercado pueden variar esa condición negativamente.

A nivel de comisiones de riego, los tres ámbitos de estudio resultaron sostenibles (Figura 4): la Comisión de Riego de Araya con 3,56, la Comisión de Riego de Potao 3,53 y la Comisión de Riego de Vinto 3,40. El mayor valor de sostenibilidad lo obtuvieron los productores de la comisión de regantes de Araya, seguido de Potao y finalmente 
Vinto. Si bien los niveles alcanzados superan el umbral mínimo establecido según la escala de Mesa y Julca (2015) y Pinedo et al. (2018), se encuentran en el rango de sostenibilidad débil, es decir, que de presentarse dificultades en los sistemas productivos por situaciones climáticas, bióticas y mercado, la sostenibilidad puede caer a niveles por debajo del umbral mínimo.

En el subindicador salud, el valor se encuentra en el umbral mínimo de sostenibilidad (UMS) (Figura 5). El valle de Pativilca cuenta con un centro de salud y tres puestos de salud con un personal médico, técnico y administrativo de 54 trabajadores, lo que evidencia una falta de equipamiento y conformación de los equipos profesionales. La calidad de los servicios de movilidad y vías de comunicación está por debajo del UMS. Según el PDSB (2015) estos dos subindicadores son claves no solo para el traslado de los bienes, sino también para el intercambio y las comunicaciones de las poblaciones.

Con respecto a los subindicadores de nivel de integración, el valor se aproxima a 4. La mayoría de los agricultores están organizados o pertenecen a alguna comisión de regantes (INEI, 2013), pero la percepción de los mismos usuarios es su escasa operatividad, y esto se refleja en la menor asistencia o capacitación que reciben (MPB, 2015).

Respecto a los subindicadores de calidad de capacitación, oferta tecnológica y conocimiento de manejo de agroquímicos, los valores están por encima de 3, y la oferta tecnológica es la más relevante, por aproximarse a 4. Según el MPB (2015), en general esta actividad es poco tecnificada, y si bien existen varias empresas que se dedican a la agroindustria, la mayor parte de la superficie cultivada está en manos de parceleros o personas naturales, que cuentan con escasa asistencia técnica. La mayor parte de la producción es para la venta, es decir, en la provincia se practica en un escaso margen la agricultura de subsistencia, y ello permite afirmar que es una de las principales actividades económicas y fuente directa de recursos de las familias identificadas en este rubro. La FAO (2016) menciona que las tecnologías adecuadas para la agricultura sostenible deben ponerse a disposición de todos los sistemas agrícolas y adaptarse a circunstancias y contextos concretos. En todos los casos, las opciones tecnológicas deben partir

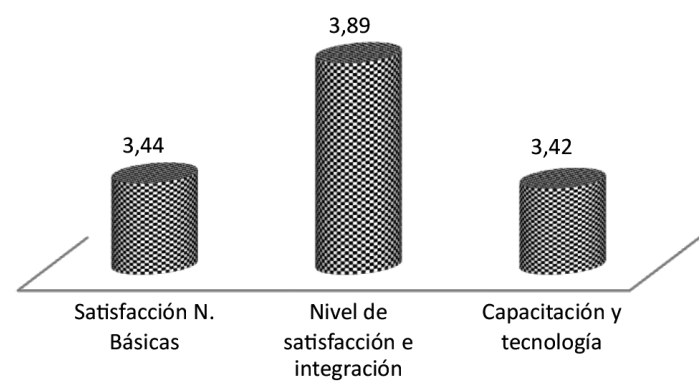

Figura 5. Valor de los indicadores de satisfacción de necesidades básicas, integración social y fortalecimiento de capacidades tecnológicas.

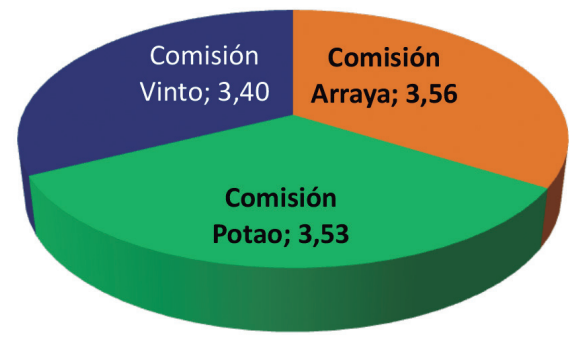

Figura 6. Niveles de IS por comisiones de regantes del valle de Pativilca.

de valoraciones sólidas del riesgo y evaluaciones de los efectos.

\section{Conclusiones}

Los indicadores de satisfacción de necesidades básicas, nivel de integración y niveles de conocimiento para los agricultores que siembran maíz amarillo duro (MAD) resultaron con valores mayores al umbral mínimo de sostenibilidad establecido.

De acuerdo a la propuesta metodológica utilizada para la producción de MAD en el valle de Pativilca, la actividad es socialmente sostenible, por tener un valor de 3,54, mayor al umbral mínimo establecido.

A nivel de comisiones de regantes, el mayor valor de Indicador Social (IS) lo alcanzó la Comisión de Riego de Araya, en segundo lugar la Comisión de Riego de Potao y tercero la Comisión de Riego de Vinto. Si i bien las tres comisiones de regantes superan el valor del umbral mínimo establecido, presentan una sostenibilidad débil y los factores bióticos, abióticos y el comportamiento del mercado pueden afectar su condición. 


\section{Literatura Citada}

ANA.

2015. Evaluación de Recursos Hídricos en la Cuenca de Pativilca. Resumen Ejecutivo. Autoridad Nacional del Agua. Lima, Perú. 53 p.

CEPES.

2018. Impacto del TLC Perú - EE.UU. en el maíz amarillo duro. Centro Peruano de Estudios Sociales. Lima, Perú. $27 \mathrm{p}$.

CIMMYT

1993. La adopción de tecnologías agrícolas: Guía para el diseño de encuestas. Programa de Economía del CIMMYT. Centro FAO. Internacional del Maíz y Trigo. México, D.F., México. 85 p.

2016. Desarrollo Agrícola Sostenible para la Seguridad Alimentaria y la Nutrición: ¿Qué función desempeña la ganadería? Un informe del Grupo de alto nivel de expertos en seguridad alimentaria y nutrición. Organización de las Naciones Unidas para la Alimentación y la Agricultura. 153 p.

Fernández, E.

2016. Impactos de los acuerdos comerciales internacionales adoptados por el Estado peruano en el cumplimiento y promoción de los derechos humanos en el Perú. Red Peruana por una Globalización con Equidad - RedGE. Lima, Perú. 26 p.

Flores, C.; Sarandón, S.

2004. Limitations of neoclassical economics for evaluating sustainability of agricultural systems: Comparing organic and conventional systems. Journal of Sustainable agricultura, 24 (2): 77-91.

Flores, C.; Sarandón, S.

2006. Desarrollo de indicadores para la evaluación de la sustentabilidad de agroecosistemas a escala regional. Revista Brasilera de Agroecología, 1 (1): 353-356.

Huamanchumo, C.

2013. La cadena de valor de maíz en el Perú: diagnóstico del estado actual, tendencias y perspectivas. IICA. 97p.

García, C.H.; Calle, L.M.

1998. Consideraciones metodológicas para la tipificación de sistemas de producción bovina a partir de fuentes secundarias. Revista Corpoica, 2(2): 6-15.

INEI.

2013. Resultados definitivos. IV Censo Nacional Agropecuario 2012. Instituto Nacional de Estadística e Informática. Perú. $62 \mathrm{p}$.

INEI.

2015. Compendio Estadístico Lima Provincias 2014. Instituto Nacional de Estadística e Informática. Lima, Perú. 565 p.
INEI.

2017. Compendio Estadístico Lima Provincias 2016. Instituto Nacional de Estadística e Informática. Lima, Perú. 567 p. Meza, Y.; Julca, A.

2015. Sustentabilidad de los sistemas de cultivo con yuca (Manihot esculenta Crantz) en la subcuenca de Santa Teresa, Cusco. Ecología Aplicada, 14 (1): 55-63.

MINAGRI.

2008. Proyecto Obras de Control y Medición de Agua por Bloques de Riego en el Valle Pativilca. Estudio de Preinversión a nivel de Perfil. Instituto Nacional de Recursos Naturales-INRENA Intendencia de Recursos Hídricos. Ministerio de Agricultura y Riego. Lima, Perú. 67 p.

MINAGRI.

2019. Observatorio de commodities: Maíz amarillo duro. Ministerio de Agricultura y Riego. Lima, Perú. 11 p. MPB.

2015. Plan de Desarrollo Concertado de la Provincia de Barranca 2015-2021. Municipalidad Provincial de Barranca. Lima, Perú. 184 p.

ONU.

2015. Sustainable Development Goals Full report of the Open Working Group of the General Assembly on is issued as document A/68/970. Organización de las Naciones Unidas. $24 \mathrm{p}$.

Pinedo R., Gómez., Julca, A.

2017. Indicadores de sostenibilidad de sistemas de producción de quinua en Chiara, Ayacucho. Aporte Santiaguino, 10 (2): 197-210.

Pinedo, R.G.; Julca A.

2018. Sostenibilidad de sistemas de producción de quinua (Chenopodium quinoa Willd.) Ecosistemas y Recursos Agropecuarios, 5(15): 399-409.

Reiche,C.; Carls J.

1996. Modelos para el desarrollo de una agricultura sostenible. COMUNIICA, 1 (3): 29-33

Sarandón, $\mathrm{S}$.

2002. El desarrollo y uso de indicadores para evaluar la sustentabilidad de los agroecosistemas. En: Sarandón, S.J. (Ed.).Agroecología: El camino hacia una agricultura sustentable. Ediciones Científicas Americanas. La Plata, Argentina. pp. 393-414.

Sarandón, S.; Zuluaga, M.; Cieza, R.; Gómez C.; Janjetic, L., Negrete, E.

2006. Evaluación de la sustentabilidad de sistemas agrícolas en fincas de Misiones, Argentina, mediante el uso de indicadores. Agroecología, 1: 19-28. 